Original article

\title{
Intestinal apicomplexan parasitoses among a hospital-based population in Honduras, 2013-2019
}

Jorge García ${ }^{1,2,3}$, Jackeline Alger ${ }^{1,3,4}$, Ramón Jeremías Soto ${ }^{2,3}$

${ }^{1}$ Servicio de Parasitología, Departamento de Laboratorio Clínico, Hospital Escuela, Tegucigalpa, Honduras

${ }^{2}$ Maestría en Epidemiología, Departamento de Salud Pública, Facultad de Ciencias Médicas, Universidad Nacional Autónoma de Honduras, Tegucigalpa, Honduras

${ }^{3}$ Instituto de Enfermedades Infecciosas y Parasitología Antonio Vidal, Tegucigalpa, Honduras

${ }^{4}$ Unidad de Investigación Científica, Facultad de Ciencias Médicas, Universidad Nacional Autónoma de Honduras, Tegucigalpa, Honduras

Introduction: Intestinal apicomplexa protozoa are a recognized cause of gastroenteritis. They are endemic in Honduras and their epidemiology varies in different population groups. Objective: To identify risk factors for cyclosporiasis, cryptosporidiosis, and cystoisosporiasis.

Materials and methods: We conducted a case-control study in a hospital-based population. We performed the diagnosis using the modified Ziehl-Neelsen staining technique and collected the information from laboratory records and clinical charts. Results: Cyclosporiasis was associated with diarrhea (OR=2.28; 95\%Cl: 1.10-4.89), weight loss (OR=12.7; 95\% Cl: 2.49-122.00), watery stools (OR=2.42; $95 \% \mathrm{Cl}: 1.26-4.65)$, and infection with another protozoan (OR=3.13; $95 \% \mathrm{Cl}$ : 1.66-5.95). Cryptosporidiosis was associated with HIV infection (OR=15.43; $95 \% \mathrm{Cl}$ : 3.34-71.22), diarrhea (OR=3.52; $95 \% \mathrm{Cl}$ : $1.40-9.40)$, lymphopenia (OR=6.16; $95 \% \mathrm{Cl}$ : 1.99-18.98), and green color stools $(\mathrm{OR}=3.00$; 95\%Cl: 1.23-7.30). Cystoisosporiasis was associated with HIV infection (OR=11.20; $95 \% \mathrm{Cl}$ : 3.53-35.44), diarrhea (OR=7.30; 95\%Cl: 1.89-28.52), leukopenia (OR=4.28; $95 \% \mathrm{Cl}: 1.33-$ $13.75)$, green color stools (OR=11.59; $95 \% \mathrm{Cl}: 1.16-558.60)$, and Charcot-Leyden crystals $(\mathrm{OR}=11.59 ; 95 \% \mathrm{Cl}: 1.16-558.60)$.

Conclusions: In this hospital-based population from Honduras, HIV infection was a risk factor for cryptosporidiosis and cystoisosporiasis, but not for cyclosporiasis.

Keywords: Parasitic diseases; intestinal diseases; cryptosporidiosis; cyclosporiasis; HIV infections; case-control studies; Honduras.

Received: $12 / 04 / 2021$

Accepted: $28 / 07 / 2021$

Published: 29/07/2021

Citation:

García JA, Alger J, Soto RJ. Intestinal apicomplexan parasitoses among a hospital-based population in Honduras, 2013-2019. Biomédica. 2021;41:734-44. https://doi.org/10.7705/biomedica.6104

\section{Corresponding author:}

Jorge García, Hospital Escuela, Boulevard Suyapa,

Calle La Salud, Tegucigalpa, Honduras

Telephone: (504) 97044797

garciaguilarjorge@gmail.com

Author contributions:

All authors contributed to the conception of the main idea of the study, the development of the research protocol, and the approval of the manuscript.

Funding:

The study was financed by the authors.

Conflicts of interest:

The authors declare that they have no conflicts of interest; the data and the results are free from plagiarism and improper manipulation. This work has not been submitted to or published in any other journal.

\section{Parasitosis por Apicomplexa intestinales en población hospitalaria en Honduras, 2013-2019}

Introducción. Los protozoos Apicomplexa intestinales son causa reconocida de gastroenteritis. Estas parasitosis son endémicas en Honduras y su epidemiologia varía según los grupos poblacionales.

Objetivo. Identificar los factores de riesgo para ciclosporiasis, criptosporidiosis y cistoisosporiasis.

Materiales y métodos. Se hizo un estudio de casos y controles en población hospitalaria. El diagnóstico se hizo utilizando la coloración modificada de Ziehl-Neelsen. La información se obtuvo del registro de laboratorio y las historias clínicas.

Resultados. La ciclosporiasis se asoció con diarrea $\left(\mathrm{OR}=2,28 ; \mathrm{IC}_{95} 1,10-4.89\right)$, pérdida de peso (OR=12,7; $\left.I C_{95 \%} 2,49-122\right)$, heces líquidas $\left(O R=2,42 ; I_{95 \%} 1,26-4,65\right)$, infección con otros protozoos $\left(\mathrm{OR}=3,13 ; \mathrm{IC}_{95 \%}, 1,66-5,95\right)$. La criptosporidiosis se asoció con el HIV $\left(\mathrm{OR}=15,43 ; \mathrm{IC}_{95 \%} 3,34-71,22\right)$, la diarrea $\left(\mathrm{OR}=3,52 ; \mathrm{IC}_{95 \%} 1,40-9,40\right)$, la linfopenia $(\mathrm{OR}=6,16$; $\left.\mathrm{IC}_{95 \%} 1,99-18,98\right)$, las heces de color verde $\left(\mathrm{OR}=3,00 ; \mathrm{IC}_{95 \%} 1,23-7,30\right)$. La cistoisosporiasis se asoció con el HIV (OR=11,20; $\left.\mathrm{IC}_{95 \%} 3,53-35,44\right)$, la diarrea $\left(\mathrm{OR}=7,30 ; \mathrm{IC}_{95 \%} 1,89-28,52\right)$, la leucopenia $\left(O R=4,28 ; I_{95 \%} 1,33-13,75\right)$, las heces de color verde $\left(O R=11,59 ; I_{95 \%} 1,16\right.$ $558,60)$, y los cristales de Charcot-Leyden (OR=11,59; $\left.I C_{95 \%} 1,16-558,60\right)$.

Conclusiones. En este estudio de base hospitalaria en Honduras, el HIV fue un factor de riesgo para la criptosporidiosis y la cistoisosporiasis, pero no así para la ciclosporiasis.

Palabras clave: enfermedades parasitarias; parasitosis intestinales; criptosporidiosis; ciclosporiasis; infecciones por HIV; estudios de casos y controles; Honduras. 
Intestinal apicomplexan protozoa (Cyclospora cayetanensis, Cryptosporidium spp., and Cystoisospora belli) are an important cause of gastroenteritis in the world, especially in low-income countries (LIC). Cyclospora cayetanensis infects subjects of all ages and its presence is associated with the rainy season and outbreaks where the transmission occurs through raw food and contaminated water (1). On the other hand, Cryptosporidium spp. is common among children less than five years old and is one of the main causes of diarrhea $(2,3)$; in the adult population, the infection is associated with immunosuppression (4). Risk factors such as poverty, lack of sanitation, contact with animals, and poor-quality water have been described (5). In most cases, $C$. belli is detected among immunosuppressed populations with HIV infection and is considered an opportunistic pathogen, although cases have also been described among immunocompetent subjects, as well as some risk factors such as lack of sanitation and poor-quality water (6).

These parasitoses are endemic in Honduras where the first reports go back to 1986 (7). Since then, they have been described several times among patients hospitalized in Hospital Escuela in Tegucigalpa (8-11), which has taken to the systematic search of the parasites in stool samples, but analytical studies are needed to better understand their local epidemiology.

We conducted the present study to identify risk factors associated with intestinal apicomplexan parasitoses and describe their distribution by age and sex, their clinical presentation, and the laboratory data among the patients from a public hospital in Honduras.

\section{Materials and methods}

We conducted a case-control study among patients attending the parasitology service at Hospital Escuela clinical laboratory in Tegucigalpa, Honduras, for six years from January, 2013, to December, 2019. Intestinal apicomplexan protozoa oocysts were identified in stool samples using the modified Ziehl-Neelsen staining technique; then, they were measured with a microscope with a micrometer eyepiece (12). Additionally, we analyzed all stool samples by direct wet smear with saline and iodine solution. The Parasitology Service has laboratory technicians, a laboratory technologist, and a parasitologist (Ph.D.) as permanent staff to carry out and supervise the parasitological diagnoses, and textbooks, bench aids, and positive samples/ slides are available for reference. The search was done in all ambulatory or hospitalized patients five or under five years of age, as well as in five-yearolds and older individuals with watery or loose stools and/or by medical request. Study subjects were also retrospectively identified from laboratory records. One stool sample was analyzed for each subject.

Cases were defined as those patients with oocysts and single apicomplexa infection whose clinical records were available at Hospital Escuela. Controls were subjects with no intestinal apicomplexa oocysts. The controls were matched by age ( \pm 2 years) and sex with the cases in a 2:1 ratio. We established three groups according to the parasite identified: Cyclospora, Cryptosporidium, or Cystoisospora.

To determine the strength of the association with possible exposure factors, we calculated odds ratios (OR) and their 95\% confidence intervals $(95 \% \mathrm{Cl})$. To evaluate the statistical significance, we used chi-square and Fisher tests while the Mann-Whitney $U$ test was used for median comparison. 
Microsoft Excel and the OpenEpi online statistical calculator were used to perform these analyzes (http://www.openepi.com/Menu/OE_Menu.htm).

The study protocol was approved by the ethics committee from the medical sciences school at Universidad Nacional Autónoma de Honduras (UNAH), (IRB 00003070 Office of Human Research Protection, https://ohrp.cit.nih. gov/search//rbDtl.aspx). We also obtained authorization from the Registration Department and the Directorate of Teaching and Research at Hospital Escuela to access the clinical records.

Our study was based on the review of laboratory records and clinical charts. There was no direct interaction with the patients and, therefore, it was not necessary to obtain informed consent or assent. Contact information (names, telephone, ID number, etc.) were not recorded to minimize a potential loss of confidentiality and each data collection instrument was assigned an identification code.

\section{Results}

From 2013 to 2019, we searched for the presence of apicomplexan protozoa in 10,938 patients; we identified 131 (1,2\%) cases of Cyclospora cayetanensis infection, 94 (0.9\%) of Cryptosporidium spp., and 50 (0.5\%) of $C$. belli. Using the data from the clinical charts available, we distributed 61 cases and 121 controls in the Cyclospora group; 53 cases and 86 controls in the Cryptosporidium group, and 27 cases and 52 controls in the Cystoisospora group. Table 1 shows the age and sex proportion between cases and controls in each group; $51.6 \%$ of cases in the Cyclospora group were male with a median age of 9.5 years; $54.7 \%$ of cases in the Cryptosporidium group were males with a median age of 4 years, and $51.9 \%$ cases in Cystoisospora group were male and the median age was 32 years. We found no statistically significant differences by sex or age among cases and controls within each group, which was expected given the study design.

Table 1. Proportional distribution of cases and controls by sex and age according to parasitosis. Hospital Escuela, Tegucigalpa, 2013-2019

\begin{tabular}{|c|c|c|c|c|c|c|}
\hline \multirow[t]{2}{*}{ Variable } & \multicolumn{2}{|c|}{ Cyclospora } & \multicolumn{2}{|c|}{ Cryptosporidium } & \multicolumn{2}{|c|}{ Cystoisospora } \\
\hline & $\begin{array}{c}\text { Cases } \\
(n=64) \\
\% \\
\end{array}$ & $\begin{array}{c}\text { Controls } \\
(n=121) \\
\%\end{array}$ & $\begin{array}{c}\text { Cases } \\
(n=53) \\
\% \\
\end{array}$ & $\begin{array}{c}\text { Controls } \\
(\mathrm{n}=86) \\
\%\end{array}$ & $\begin{array}{c}\begin{array}{c}\text { Cases } \\
(\mathrm{n}=27) \\
\%\end{array} \\
\end{array}$ & $\begin{array}{c}\text { Controls } \\
(n=52) \\
\%\end{array}$ \\
\hline \multicolumn{7}{|l|}{ Sex } \\
\hline Male & 51.6 & 48.8 & 54.7 & 65.1 & 51.9 & 57.7 \\
\hline Female & 48.4 & 51.2 & 45.3 & 34.9 & 48.1 & 42.3 \\
\hline Chi square & \multicolumn{2}{|c|}{$p=0.8352$} & \multicolumn{2}{|c|}{$p=0.2988$} & \multicolumn{2}{|c|}{$p=0.7973$} \\
\hline \multicolumn{7}{|l|}{ Age (years) } \\
\hline$<1$ & 1.5 & 5.8 & 18.9 & 26.7 & 3.7 & 5.8 \\
\hline $1-5$ & 43.8 & 41.3 & 37.7 & 43.0 & 3.7 & 1.9 \\
\hline $6-15$ & 9.4 & 14.0 & 5.7 & 3.6 & 0.0 & 1.9 \\
\hline$>15$ & 45.3 & 38.8 & 37.7 & 26.7 & 92.6 & 90.4 \\
\hline Median & 9.5 & 8.0 & 4 & 2.5 & 32.0 & 36 \\
\hline (IR) & $(3.0-23.5)$ & $(2.0-24.0)$ & $(1.0-34.0)$ & $(0.8-20.7)$ & $(27.0-45.0)$ & $(30-47.2)$ \\
\hline Mann- Whitney U & \multicolumn{2}{|c|}{$p=0.6213$} & \multicolumn{2}{|c|}{$p=0.7840$} & \multicolumn{2}{|c|}{$p=0.6423$} \\
\hline
\end{tabular}


Table 2 describes the variables in the Cyclospora group. The main differences observed between cases and controls were living in a house with concrete floors ( $55.2 \%$ vs. $30.8 \%$ ), adobe walls ( $44.8 \%$ vs. $10.3 \%)$, block or brick walls $(51.7 \%$ vs. $71.8 \%)$, metal roof $(62.1 \%$ vs.72.2\%), piped water service ( $77.8 \%$ vs. $83.8 \%)$, sewerage service $(53.6 \%$ vs. $71.8 \%)$, and having a dog in the house $(54.2 \%$ vs. $38.9 \%)$. The main differences in the clinical presentation were diarrhea ( $75.5 \%$ vs. $57.4 \%)$, abdominal pain ( $42.9 \%$ vs. $34.7 \%)$, weight loss $(20.4 \%$ vs. $2.0 \%)$, HIV infection ( $14.0 \%$ vs. $4.6 \%)$, leukocytosis $(18.9 \%$ vs. $30.5 \%$ ), neutrophilia (16.7\% vs. $32.5 \%$ ), thrombocytosis (30.6\% vs. $13.4 \%)$, watery stools ( $42.2 \%$ vs. $23.1 \%)$, fat visible under the microscope $(14.1 \%$ vs. $4.9 \%)$, and infection with other protozoa (53.1\% vs. $26.4 \%)$.

Blastocystis spp. was the most frequently identified parasite (37.7\% vs $19.8 \%)$. The participants with a significant probability of being diagnosed or suffering from cyclosporiasis were those living in houses with concrete floor (OR=2.77; 95\%IC: $1.02-7.52)$ and adobe walls $(\mathrm{OR}=7.1 ; 95 \% \mathrm{Cl}: 2.0-$ $25.24)$, as well as those with diarrhea (OR=2.28; $95 \% \mathrm{Cl}$ : $1.10-4.89)$, weight loss (OR=12.7; 95\%Cl: $2.49-122)$, thrombocytosis (OR=2.84; $95 \% \mathrm{Cl}: 1.10$ 7.36), watery stools $(\mathrm{OR}=2.42 ; 95 \% \mathrm{Cl}: 1.26-4.65)$, and coinfection with other protozoa (OR=3.13; $95 \% \mathrm{Cl}: 1.66-5.95)$. On the contrary, subjects who lived in houses with brick walls (OR=0.31; 95\% Cl: $0.11-0.86)$ and had vomiting (OR $=0.48 ; 95 \% \mathrm{Cl}: 0.23-0.97$ ) showed a significantly lower probability of being diagnosed with cyclosporiasis.

In the Cryptosporidium group (table 3), the main differences between cases and controls were living in a house with concrete floors $(43.5 \%$ vs. $21.9 \%)$, block or brick walls $(52.2 \%$ vs. $58.1 \%)$, metal roof $(59.1 \%$ vs. $65.6 \%)$, piped water service (69.9\% vs. $90.0 \%)$, sewerage service $(60.0 \%$ vs. $57.1 \%)$, and having a dog in the house (39.1\% vs. $29.2 \%)$. As for the clinical presentation, the main differences between cases and control were diarrhea ( $80.4 \%$ vs. $53.8 \%)$, fever (65.2\% vs. $51.3 \%)$, cough ( $21.7 \%$ vs. $15.4 \%)$, HIV infection (30.0\% vs. $2.7 \%$ ), leukopenia (37.5\% vs. $9.7 \%)$, lymphopenia (35.9\% vs. $8.3 \%)$, anemia ( $65.0 \%$ vs. $53.2 \%)$, green color stools $(28.3 \%$ vs. $11.6 \%)$, loose stools ( $24.5 \%$ vs. $33.7 \%$ ), watery stools ( $28.3 \%$ vs. $20.9 \%)$, stools with mucus $(52.8 \%$ vs. $30.2 \%)$, and the presence of Charcot-Leyden crystals (3.8\% vs. $1.2 \%)$. Subjects with a significantly higher probability of being diagnosed or suffering from cryptosporidiosis were those with HIV infection $(\mathrm{OR}=15.43 ; 95 \% \mathrm{Cl}$ : 3.34-71.22), a history of diarrhea (OR=3.52; $95 \% \mathrm{Cl}$ : 1.40-9.40), leukopenia (OR=5.60; $95 \% \mathrm{Cl}: 1.90-16.12)$, lymphopenia $(\mathrm{OR}=6.16$; $95 \% \mathrm{Cl}: 1.99-18.98)$, green color stools (OR=3.00; $95 \% \mathrm{Cl}: 1.23-7.30)$, and stools with mucus (OR=2.58; 95\% $\mathrm{Cl}: 1.27-5.25)$.

In the Cystoisospora group (table 4), we were not able to collect data about house characteristics. The main differences between cases and controls were diarrhea ( $87.5 \%$ vs. $48.8 \%$ ), vomit ( 58.3 vs. $39.0 \%)$, abdominal pain $(41.7 \%$ vs. $19.5 \%$ ), HIV infection ( $77.8 \%$ vs. $23.8 \%$ ), leukopenia ( $43.5 \%$ vs. $16.3 \%)$, lymphopenia (52.2\% vs. $15.7 \%)$, and anemia (78.3\% vs. $51.2 \%)$. Cases presented with stools of green color ( $18.5 \%$ vs. $1.9 \%)$, watery stools $(44.4 \%$ vs. $30.8 \%)$, mucus $(48.1 \%$ vs. $44.2 \%)$, fat visible under the microscope $(18.5 \%$ vs. $1.9 \%$ ), and Charcot-Leyden crystals ( $18.5 \%$ vs. $1.9 \%$ ) more frequently than controls. Subjects with the most statistically significant probability of being diagnosed or suffering from cystoisosporiasis were those with HIV infection (OR=11.20; 95\% Cl: 3.53-35.44), diarrhea (OR=7.30; 95\%Cl: 1.89-28.52), leukopenia (OR=4.28; 95\%Cl: 1.33-13.75), anemia (OR=3.43; 95\%Cl: 1.08 $10.93)$, green color stools ( $\mathrm{OR}=11.59 ; 95 \% \mathrm{Cl}: 1.16-558.60)$, microscopically 
visible fat $(\mathrm{OR}=11.59 ; 95 \% \mathrm{Cl}: 1.16-558.60)$, and the presence of CharcotLeyden crystals $(\mathrm{OR}=11.59 ; 95 \% \mathrm{Cl}$ : 1.16-558.60). Subjects with leukocytosis had a significantly lower probability of suffering cystoisosporiasis $(\mathrm{OR}=0.12$; 95\%Cl: 0.003-0.92).

Table 2. Characteristics of cyclosporiasis cases and their controls, Hospital Escuela, Tegucigalpa, 2013-2019

\begin{tabular}{|c|c|c|c|c|c|}
\hline Variable & $\begin{array}{l}\text { Cases } \\
\mathrm{N}=64 \\
\mathrm{n}(\%)\end{array}$ & $\begin{array}{c}\text { Controls } \\
\mathrm{N}=121 \\
\mathrm{n}(\%)\end{array}$ & OR & $\mathrm{Cl} 195 \%$ & $\mathbf{p}$ \\
\hline \multicolumn{6}{|l|}{ House building materials } \\
\hline Floor & $n=29$ & $\mathrm{n}=39$ & & & \\
\hline Concrete & $16(55.2)$ & $12(30.8)$ & 2.77 & $1.02-7.52$ & 0.0762 \\
\hline Brick & $9(31.0)$ & $23(59.0)$ & 0.31 & $0.11-0.86$ & 0.0416 \\
\hline Soil & $4(13.8)$ & $4(10.3)$ & 1.40 & $0.23-8.24$ & $0.9355^{*}$ \\
\hline Walls & $\mathrm{n}=29$ & $\mathrm{n}=39$ & & & \\
\hline Block/Brick & $15(51.7)$ & $28(71.8)$ & 0.42 & $0.15-1.15$ & 0.1490 \\
\hline Adobe & $13(44.8)$ & $4(10.3)$ & 7.10 & $2.0-25.24$ & 0.0029 \\
\hline Roof & $\mathrm{n}=29$ & $\mathrm{n}=36$ & & & \\
\hline Metal & $18(62.1)$ & $26(72.2)$ & 0.63 & $0.22-1.79$ & 0.5463 \\
\hline Tile & $9(31.0)$ & 7 (19.4) & 1.80 & $0.59-5.83$ & 0.4303 \\
\hline Water source & $\mathrm{n}=27$ & $\mathrm{n}=37$ & & & \\
\hline Pipe service & $21(77.8)$ & 31 (83.8) & 0.67 & $0.19-2.38$ & 0.7766 \\
\hline Others (well, river, etc.) & $6(22.2)$ & $6(16.2)$ & 1.47 & $0.42-5.20$ & 0.7766 \\
\hline Sewerage & $\mathrm{n}=28$ & $n=39$ & & & \\
\hline Sewerage & $15(53.6)$ & $28(71.8)$ & 0.45 & $0.16-1.25$ & 0.1547 \\
\hline Latrine & 12 (42.9) & $10(25.6)$ & 2.10 & $0.77-6.13$ & 0.2023 \\
\hline None & $1(3.6)$ & $1(2.6)$ & 1.40 & $0.02-113.3$ & $>0.999^{*}$ \\
\hline Origin & $\mathrm{n}=61$ & $\mathrm{n}=116$ & & & \\
\hline Tegucigalpa city & 39 (63.9) & $74(63.8)$ & 1.00 & $0.53-1.92$ & 0.8839 \\
\hline Francisco Morazán & 7 (11.5) & $15(12.9)$ & 0.87 & $0.33-2.27$ & 0.9687 \\
\hline $\begin{array}{l}\text { Other departments } \\
\text { Animal contact }\end{array}$ & $\begin{array}{c}15(24.6) \\
n=24\end{array}$ & $\begin{array}{c}27(23.3) \\
n=36\end{array}$ & 1.07 & $0.52-2.21$ & 0.9925 \\
\hline Dog & $13(54.2)$ & $14(38.9)$ & 1.85 & $0.65-5.28$ & 0.3679 \\
\hline Chicken & $5(20.8)$ & $6(16.7)$ & 1.30 & $0.27-5.98$ & $0.9341^{*}$ \\
\hline Cat & $5(20.8)$ & $6(16.7)$ & 1.30 & $0.27-5.98$ & $0.9341^{*}$ \\
\hline Clinical presentation & $n=49$ & $\mathrm{n}=101$ & & & \\
\hline Diarrhea & $37(75.5)$ & $58(57.4)$ & 2.28 & $1.10-4.89$ & 0.0482 \\
\hline Fever & $19(38.8)$ & $55(54.5)$ & 0.52 & $0.26-1.06$ & 0.1037 \\
\hline Vomit & $17(34.7)$ & $53(52.5)$ & 0.48 & $0.23-0.97$ & 0.0611 \\
\hline Abdominal pain & $21(42.9)$ & $35(34.7)$ & 1.41 & $0.70-2.84$ & 0.4271 \\
\hline Weight loss & $10(20.4)$ & $2(2.0)$ & 12.7 & $2.49-122.00$ & $0.0005^{\star}$ \\
\hline Immunosuppression & $\mathrm{n}=57$ & $n=108$ & & & \\
\hline HIV & $8(14.0)$ & $5(4.6)$ & 3.36 & $0.90-13.60$ & $0.0729^{*}$ \\
\hline Cancer & 7 (12.3) & $5(4.6)$ & 2.80 & $0.74-12.05$ & $0.1433^{*}$ \\
\hline $\begin{array}{l}\text { Hematological malignancy } \\
\text { Blood cell count }\end{array}$ & $\begin{array}{l}8(14.0) \\
n=38\end{array}$ & $\begin{array}{l}9(8.3) \\
n=83\end{array}$ & 1.79 & $0.65-4.94$ & 0.3808 \\
\hline Leukocytosis & $7(18.9)$ & $25(30.5)$ & 0.52 & $0.20-1.34$ & 0.2584 \\
\hline Leukopenia & $4(10.8)$ & $10(12.2)$ & 0.87 & $0.18-3.31$ & $>0.9999^{*}$ \\
\hline Neutrophilia & $6(16.7)$ & $27(32.5)$ & 0.39 & $0.15-1.04$ & 0.0893 \\
\hline Neutropenia & 5 (13.9) & $7(8.4)$ & 1.75 & $0.40-6.94$ & $0.5497^{\star}$ \\
\hline Lymphocytosis & $4(11.1)$ & $6(7.4)$ & 1.56 & $0.30-7.08$ & $0.7362^{*}$ \\
\hline Lymphopenia & 5 (13.9) & $17(21.0)$ & 0.58 & $0.19-1.73$ & 0.4743 \\
\hline Anemia & $21(55.3)$ & $44(53.0)$ & 1.09 & $0.50-2.36$ & 0.9728 \\
\hline Thrombocytosis & $11(30.6)$ & $11(13.4)$ & 2.84 & $1.10-7.36$ & 0.0518 \\
\hline Stool characteristics & $n=64$ & $n=121$ & & & \\
\hline Formed consistency & $9(14.1)$ & $25(20.7)$ & 0.62 & $0.27-1.44$ & 0.3667 \\
\hline Soft consistency & 12 (18.8) & $31(25.6)$ & 0.67 & $0.32-1.41$ & 0.3847 \\
\hline Loose consistency & $16(25.0)$ & $37(30.6)$ & 0.75 & $0.38-1.50$ & 0.5304 \\
\hline Watery consistency & 27 (42.2) & $28(23.1)$ & 2.42 & $1.26-4.65$ & 0.0115 \\
\hline Mucus & $14(21.9)$ & $39(32.2)$ & 0.58 & $0.29-1.19$ & 0.1901 \\
\hline Leukocytes & $10(15.6)$ & $25(20.7)$ & 0.71 & $0.32-1.59$ & 0.5257 \\
\hline Fat under the microscope & $9(14.1)$ & $6(4.9)$ & 3.13 & $1.06-9.25$ & 0.0608 \\
\hline Charcot-Leyden crystals & $2(3.1)$ & 4 (3.3) & 0.94 & $0.08-6.79$ & $>0.9999^{*}$ \\
\hline Other protozoa & $34(53.1)$ & $32(26.4)$ & 3.15 & $1.66-5.95$ & 0.0006 \\
\hline
\end{tabular}


Table 3. Characteristics of cryptosporidiosis cases and their controls. Hospital Escuela, Tegucigalpa, 2013-2019

\begin{tabular}{|c|c|c|c|c|c|}
\hline Variable & $\begin{array}{l}\text { Cases } \\
\mathrm{N}=53 \\
\mathrm{n}(\%)\end{array}$ & $\begin{array}{c}\text { Controls } \\
\mathrm{N}=86 \\
\mathrm{n}(\%)\end{array}$ & OR & $\mathrm{Cl} 95 \%$ & $\mathbf{p}$ \\
\hline \multicolumn{6}{|l|}{ House materials building } \\
\hline Floor & $n=23$ & $n=32$ & & & \\
\hline Concrete & $10(43.5)$ & $7(21.9)$ & 2.74 & $0.85-8.9$ & 0.1574 \\
\hline Brick & $5(21.7)$ & $16(50.0)$ & 0.28 & $30.08-0.93$ & 0.0648 \\
\hline Soil & $7(30.4)$ & $9(28.1)$ & 1.10 & $0.34-3.62$ & 0.9085 \\
\hline Walls & $n=23$ & $n=31$ & & & \\
\hline Block/Brick & $12(52.2)$ & $18(58.1)$ & 0.78 & $0.26-2.33$ & 0.8777 \\
\hline Adobe & $8(34.8)$ & $10(32.2)$ & 1.12 & $0.35-3.50$ & 0.9225 \\
\hline Roof & $\mathrm{n}=22$ & $n=32$ & & & \\
\hline Metal & $13(59.1)$ & $21(65.6)$ & 0.75 & $0.24-2.31$ & 0.8401 \\
\hline Tile & 4 (18.2) & $6(18.7)$ & 0.96 & $0.17-4.76$ & $>0.9999^{*}$ \\
\hline Water source & $n=23$ & $n=30$ & & & \\
\hline Pipe service & $16(69.6)$ & $27(90.0)$ & 0.25 & $0.04-1.34$ & $0.1266^{\star}$ \\
\hline Others (well, river, etc.) & $7(30.4)$ & $3(10.0)$ & 3.94 & $0.89-17.42$ & $0.1266^{*}$ \\
\hline Sewerage & $\mathrm{n}=20$ & $n=28$ & & & \\
\hline Sewerage & $12(60.0)$ & $16(57.1)$ & 1.12 & $0.35-3.61$ & 0.9212 \\
\hline Latrine & $6(30.0)$ & $12(42.9)$ & 0.57 & $0.17-1.92$ & 0.3453 \\
\hline Origin & $\mathrm{n}=49$ & $\mathrm{n}=85$ & & & \\
\hline Tegucigalpa city & $26(53.1)$ & $58(68.2)$ & 0.52 & $0.25-1.08$ & 0.1179 \\
\hline Francisco Morazán & $8(16.3)$ & $10(11.8)$ & 1.46 & $0.53-3.99$ & 0.6292 \\
\hline Other departments & $15(30.6)$ & $17(20.0)$ & 1.76 & $0.78-3.95$ & 0.2397 \\
\hline Animal contact & $n=23$ & $n=24$ & & & \\
\hline Dog & $9(39.1)$ & $7(29.2)$ & 1.50 & $0.46-5.26$ & 0.6798 \\
\hline Chicken & $8(34.8)$ & $5(20.8)$ & 2.00 & $0.54-7.48$ & 0.4578 \\
\hline Cat & $1(4.3)$ & $4(16.7)$ & 0.22 & $0.004-2.62$ & $0.3741^{\star}$ \\
\hline Clinical presentation & $n=46$ & $\mathrm{n}=78$ & & & \\
\hline Diarrhea & $37(80.4)$ & $42(53.8)$ & 3.52 & $1.40-9.40$ & 0.0054 \\
\hline Fever & $30(65.2)$ & 40 (51.3) & 1.78 & $0.84-3.78$ & 0.1856 \\
\hline Vomit & $20(43.5)$ & $36(46.2)$ & 0.89 & $0.43-1.86$ & 0.9184 \\
\hline Abdominal pain & $10(21.7)$ & $16(20.5)$ & 1.07 & $0.44-2.62$ & 0.9471 \\
\hline Cough & $10(21.7)$ & $12(15.4)$ & 1.50 & $0.60-3.80$ & 0.5148 \\
\hline Immunosuppression & $\mathrm{n}=50$ & $\mathrm{n}=74$ & & & \\
\hline HIV & $15(30.0)$ & $2(2.7)$ & 15.43 & $3.34-71.22$ & 0.00005 \\
\hline Cancer & $5(10.0)$ & $3(4.1)$ & 2.63 & $0.48-17.62$ & $0.3419^{*}$ \\
\hline Hematological malignancy & $5(10.0)$ & $5(6.8)$ & 1.53 & $30.33-7.05$ & $0.7420^{*}$ \\
\hline Blood cell count & $n=40$ & $n=62$ & & & \\
\hline Leucopenia & $15(37.5)$ & $6(9.7)$ & 5.60 & $1.9-16.12$ & 0.0016 \\
\hline Leukocytosis & $10(25.0)$ & $19(30.6)$ & 0.75 & $0.31-1.84$ & 0.6948 \\
\hline Neutrophilia & $5(13.2)$ & $16(26.7)$ & 0.42 & $0.14-1.25$ & 0.1703 \\
\hline Neutropenia & $5(13.2)$ & $7(11.7)$ & 1.14 & $0.26-4.59$ & $>0.9999^{*}$ \\
\hline Lymphocytosis & $12(30.8)$ & $8(13.3)$ & 2.89 & $1.05-7.90$ & 0.0617 \\
\hline Lymphopenia & $14(35.9)$ & $5(8.3)$ & 6.16 & $1.99-18.98$ & 0.0016 \\
\hline Anemia & $26(65.0)$ & $33(53.2)$ & 1.60 & $0.71-3.70$ & 0.3319 \\
\hline Thrombocytosis & $8(20.0)$ & $14(22.9)$ & 0.83 & $0.32-2.23$ & 0.9499 \\
\hline Stool characteristics & $n=53$ & $\mathrm{n}=86$ & & & \\
\hline Green color & $15(28.3)$ & $10(11.6)$ & 3.00 & $1.23-7.30$ & 0.0239 \\
\hline Formed consistency & $4(7.5)$ & $11(12.8)$ & 0.55 & $1.16-1.84$ & 0.4925 \\
\hline Soft consistency & $21(39.6)$ & $28(32.6)$ & 1.35 & $0.66-2.76$ & 0.5067 \\
\hline Loose consistency & $13(24.5)$ & 29 (33.7) & 0.63 & $0.29-1.37$ & 0.3390 \\
\hline Watery consistency & 15 (28.3) & $18(20.9)$ & 1.49 & $0.67-3.29$ & 0.5853 \\
\hline Mucus & $28(52.8)$ & $26(30.2)$ & 2.58 & $1.27-5.25$ & 0.0132 \\
\hline Leukocytes & $11(20.7)$ & $20(23.2)$ & 0.86 & $0.37-1.98$ & 0.8932 \\
\hline Fat under the microscope & $4(7.5)$ & $13(15.1)$ & 0.45 & $0.14-1.48$ & 0.2923 \\
\hline Charcot-Leyden crystals & $2(3.8)$ & $1(1.2)$ & 3.33 & $0.16-198.90$ & $0.6481^{*}$ \\
\hline Other protozoa & $7(13.2)$ & $16(18.6)$ & 0.53 & $0.18-1.58$ & 0.5507 \\
\hline
\end{tabular}


Table 4. Characteristics of cystoisosporosis cases and their controls. Hospital Escuela, Tegucigalpa, 2013-2019

\begin{tabular}{|c|c|c|c|c|}
\hline Variable & $\begin{array}{c}\text { Cases } \\
\mathrm{N}=27 \\
\mathrm{n}(\%) \\
\end{array}$ & $\begin{array}{c}\text { Controls } \\
\begin{array}{l}\mathrm{N}=52 \\
\mathrm{n}(\%)\end{array}\end{array}$ & $\mathrm{Cl} 95 \%$ & p \\
\hline Origin & $\mathrm{n}=26$ & $n=49$ & & \\
\hline Tegucigalpa city & $17(65.4)$ & $23(46.9)$ & $2.130 .79-5.71$ & 0.2006 \\
\hline Francisco Morazán & $2(7.7)$ & $7(14.3)$ & $0.500 .05-2.94$ & $0.6640^{\star}$ \\
\hline Other departments & $7(26.9)$ & $19(38.8)$ & $0.580 .20-1.64$ & 0.4404 \\
\hline Animal contact & $\mathrm{n}=6$ & $n=7$ & & \\
\hline Dog & $3(50.0)$ & $3(42.9)$ & $1.330 .09-9.13$ & $>0.999^{*}$ \\
\hline Chicken & $1(16.7)$ & $3(42.9)$ & $0.270 .004-5.49$ & $0.6853^{*}$ \\
\hline Cat & $1(16.7)$ & $1(14.3)$ & $1.200 .012-9.70$ & $>0.999^{\star}$ \\
\hline Clinical presentation & $\mathrm{n}=24$ & $n=41$ & & \\
\hline Diarrhea & $21(87.5)$ & 8.8) & $7.301 .89-28.52$ & 0.004 \\
\hline Fever & $(45.8)$ & 17( & $1.190 .43-3.29$ & 0.9332 \\
\hline Vomit & $(58.3)$ & 16( & $2.200 .78-6.10$ & 0.2120 \\
\hline Abdominal pain & $10(41.7)$ & & $2.900 .96-9.03$ & 0.1012 \\
\hline Immunosuppression & $\mathrm{n}=27$ & $n-12$ & & \\
\hline HIV & $21(77.8)$ & $10(23.8)$ & $11.203 .53-35.44$ & 0.00003 \\
\hline Cancer & $1(3.7)$ & $2(4.7)$ & $0.760 .02-15.64$ & $>0.9999^{\star}$ \\
\hline Hematological malignancy & $1(3.7)$ & $4(9.5)$ & $0.370 .007-4.02$ & $0.6892^{*}$ \\
\hline Blood cell count & $n=23$ & & & \\
\hline Leu & & & -0.92 & $0.0380^{*}$ \\
\hline Leuk & 10 (1) & & 4.281 .3 & \\
\hline Neutro & & $13(3$ & $0.200 .04-1.02$ & 0.0927 \\
\hline Neutr & $2(9.1)$ & $3(7.5)$ & $1.230 .09-11.68$ & $0.9999^{*}$ \\
\hline Lymp & $1(4.3)$ & $2(2.4)$ & $0.860 .01-17.53$ & $0.99999^{*}$ \\
\hline Lymphopenia & $12(52.2)$ & $13(15.7)$ & $2.260 .79-6.48$ & 0.1377 \\
\hline Anemia & $18(78.3)$ & $22(51.2)$ & $3.431 .08-10.93$ & 0.0597 \\
\hline Thromboc & $4(18.2)$ & $5(12.2)$ & $1.600 .27-8.41$ & $0.7688^{*}$ \\
\hline Stool c & & & & \\
\hline Greer & & ) & $11.591 .16-558.60$ & $0.0323^{*}$ \\
\hline sistency & 1 & & $0.004-1.28$ & $99^{*}$ \\
\hline Soft c & 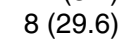 & 6( & $3.220 .83-12.77$ & $0.0960^{*}$ \\
\hline Loos & 2) & $21(4$ & $0.420 .14-1.22$ & 0.1727 \\
\hline onsistency & $12(44.4)$ & $16(30.8)$ & $1.800 .68-4.70$ & 0.3384 \\
\hline Mucu & $13(48.1)$ & $23(44.2)$ & $1.170 .46-2.97$ & 0.9255 \\
\hline Leukocyte & $4(14.8)$ & $10(25.0)$ & $0.520 .15-1.79$ & 0.8596 \\
\hline Fat ur & $5(18.5)$ & $1(1.9)$ & $11.591 .16-558.60$ & $0.0320^{*}$ \\
\hline Charcot-Leyden crystals & $5(18.5)$ & $1(1.9)$ & $11.591 .16-558.60$ & $0.0323^{*}$ \\
\hline Other protozoa & $5(18.5)$ & $15(28.8)$ & $0.560 .17-1.75$ & 0.4663 \\
\hline
\end{tabular}

${ }^{*}$ Fisher test

\section{Discussion}

Some of the risk factors for these parasitoses were poverty and lack of drinking water and sewerage services (5,6,13-15). These conditions are prevalent in Honduras and they may explain our findings. Living in a house with brick floors might protect against cyclosporiasis (and possibly against cryptosporidiosis), which may be a proxy indicator of better socioeconomic status. On the other hand, living in a house with mud walls was associated with cyclosporiasis, which might indicate lower socioeconomic status but was not measured in this study. We observed differences in the frequency of piped water service, sewerage, and the presence of domestic animals between cases and controls in the C. cayetanensis and Cryptosporidium spp. groups. However, these findings were not statistically significant.

We found an association between cyclosporiasis, diarrhea, and weight loss. These results are consistent with the clinical presentations described for cyclosporiasis in other studies including diarrhea, abdominal pain, fatigue, weight loss, and vomiting both in children and adults $(16,17)$. Watery stools and the presence of other protozoans were associated with cyclosporiasis 
in this study. Similar observations have been made in Honduras relating watery stools to the clinical presentation (11) and the presence of other protozoa coinfections in other studies (16) probably explained by the similar transmission modes of $C$. cayetanensis and other commensal protozoans.

Diarrhea was associated with Cryptosporidium cases, which is consistent with other studies (17-19). The proportion of cough was higher among the cases $(21.7 \%)$ compared to the controls $(15.4 \%)$ in the Cryptosporidium group while respiratory symptoms were not frequent among cyclosporiasis and cystoisosporiasis cases. Studies in Uganda showed that coughing was present in $78 \%$ of immunocompetent children with cryptosporidiosis while oocysts were identified in the sputum of $35.4 \%$ of children with cough (20). In adults, respiratory cryptosporidiosis was present in $1.3 \%$ of subjects with HIV infection and $4.4 \%$ of HIV-negative individuals with suspected tuberculosis (21). In our study, coughing was not statistically associated with cryptosporidiosis but the results could point to a possible respiratory infection. Unfortunately, the systematic search for this parasite was not conducted despite the presence of coughing.

In our study, diarrhea was associated with $C$. belli cases and we observed that fever, vomiting, and abdominal pain were frequent among cases but with no statistical association, which is consistent with the clinical presentation already described (6): diarrhea with abdominal pain, nausea, fever, and weight loss.

We found that subjects with HIV infection were 15 times more likely to be infected by Cryptosporidium spp. and 11 times by C. belli but HIV infection was not a risk factor for $C$. cayetanensis. These findings are consistent with observations previously made in Honduras where Cryptosporidium spp. and C. belli were more frequent among HIV-infected populations but was not the case of $C$. cayetanensis (10). Similar observations have been reported in other countries where Cryptosporidium spp. and C. belli were associated with immunosuppressed people due to HIV, organ transplants, or leukemia (22-25). Our results confirmed that these parasitoses are opportunistic and endemic in immunosuppressed populations, especially those infected with HIV. Thus, the systematic surveillance of these protozoans is highly recommended. Leukopenia and lymphopenia were associated with cryptosporidiosis. Similarly, we found an association with leukopenia and cystoisosporiasis but not with lymphopenia, although it was more frequent among the cases $(43.5 \%)$ than the controls (16.3\%) probably due to immunosuppression by HIV infection, which was frequent among these cases.

We found no association between cryptosporidiosis and stool consistency. On the other hand, a formed consistency of the stools was present in $47 \%$ of cases probably because of asymptomatic oocysts shedding after the episode of diarrhea, which in cryptosporidiosis can occur for up to seven months (26). It is possible that among our participants, the episode of diarrhea resolved while the asymptomatic period of oocyst shedding remained. Charcot-Leyden crystals observed microscopically in the stool examination were associated with cystoisosporiasis but no association with eosinophilia was found, which would have been expected because these crystals are related to the activity of eosinophils. Some studies have reported cystoisosporiasis associated with eosinophilia and Charcot-Leyden crystals (27).

Limitations in our study refer to the diagnostic method. The modified ZiehlNeelsen staining technique is a cheap and relatively simple laboratory method, 
although it requires quality reagents, equipment, and human resources, but it is not very sensitive for detecting the infections given the different patterns of parasite excretion or the low density in the stool sample. It has been estimated that this staining technique can detect $78 \%$ of cases compared to other more expensive and specialized methods (fluorescence microscopy, immunoenzymatic assays, or molecular biology) (14,27-31). This could have affected the number of cases detected during this period or, more importantly, the selection of controls. Therefore, the inclusion of false negative controls should not be discarded and, thus, selection bias. Ideally, the use of molecular tools with greater sensitivity is required to detect these infections. Secondly, our sample size represented $51.3 \%$ of the total cases in the 2013-2019 period. Besides, the information on the variables was not complete, which reflected in the wide $95 \%$ confidence intervals estimated for the ORs and indicates low precision and statistical power, which should be considered when interpreting the results. Thirdly, we did not search for other non-parasitic pathogens causing diarrhea, so we do not know if the cases or the controls had infections other than the parasitoses under study. Additionally, we could not determine if the clinical presentation and laboratory data were risk factors since they were not defined as exposure variables occurring before the parasitosis.

In conclusion, HIV infection was an important risk factor for acquiring Cryptosporidium spp. and C. belli, but not $C$. cayetanensis among this hospital-based population in Honduras. Diarrhea is part of the clinical presentation of the three parasitoses, but it was only associated with cyclosporiasis. We also found associations with other variables but the study's low statistical power prevents further conclusions. However, such variables may be risk factors for acquiring these parasitoses, and, therefore, it would be convenient to study them using laboratory detection tools with a higher sensitivity in cohort studies. Although these parasites are endemic in Honduras, further studies are required to better understand the local epidemiology, clinical presentation, circulating Cryptosporidium species, and other aspects yet to be explored.

\section{Acknowledgements}

We want to thank the laboratory technicians Wendy López and Mercedes Godoy from the parasitology service at Hospital Escuela for the microscopic diagnosis and the staff from the surveillance and registration departments for their support in the search of clinical records. This research is the result of JG's thesis work in the Master's Program in Epidemiology, Department of Public Health, Faculty of Medical Sciences at Universidad Nacional Autónoma de Honduras (UNAH); JA and RJS were thesis advisers.

\section{References}

1. Li J, Wang R, Chen Y, Xiao L, Zhang L. Cyclospora cayetanensis infection in humans: Biological characteristics, clinical features, epidemiology, detection method and treatment. Parasitology. 2020;147:160-70. https://doi.org/10.1017/S0031182019001471

2. Platss-Mills J, Babji S, Bodhidatta L, Gratz J, Haque R, Havt A, et al. Pathogen-specific burdens of community diarrhoea in developing countries: a multisite birth cohort study (MALED). Lancet Glob Health. 2015;3:e564-75. https://doi.org/10.1016/S2214-109X(15)00151-5

3. Kotloff KL, Nataro JP, Blackwelder WC, Nasrin D, Farag TH, Panchalingam S, et al. Burden and aetiology of diarrhoeal disease in infants and young children in developing countries (the Global Enteric Multicenter Study, GEMS): A prospective, case-control study. Lancet. 2013;382:209-22. https://doi.org/10.1016/S0140-6736(13)60844-2 
4. Dillingham R, Pinkerton R, Leger P, Severe P, Guerrant RL, Pape JW, et al. High early mortality in patients with chronic acquired immunodeficiency syndrome diarrhea initiating antiretroviral therapy in Haiti: A case-control study. Am J Trop Med Hyg. 2009;80:1060-4.

5. Bouzid M, Kintz E, Hunter PR. Risk factors for Cryptosporidium infection in low and middle income countries: A systematic review and meta-analysis. PLoS Negl Trop Dis. 2018;12:e0006553. https://doi.org/10.1371/journal.pntd.0006553

6. Dubey JP, Almeria S. Cystoisospora belli infections in humans: the past 100 years. Parasitology. 2019;146:1490-527. https://doi.org/10.1017/S0031182019000957

7. Kaminsky R, Canales M. Criptosporidiosis en Honduras. Rev Méd Hondur. 1986;54:268-77.

8. Kaminsky R. Isospora belli en Honduras. Parasitologia Dia. 1990;14:73-8.

9. Kaminsky R. Cyclospora cayetanensis: nuevo apicomplexa intestinal, actualización y presentación de casos en el Hospital Escuela. Rev Méd Hondur. 1997;65:68-72.

10. Kaminsky, RG. Comparación epidemiológica de apicomplexa intestinales en el Hospital Escuela, Honduras. Rev Méd Hondur. 2002;70:164-72.

11. Kaminsky RG, Lagos J, Raudales-Santos G, Urrutia S. Marked seasonality of Cyclospora cayetanensis infections: Ten-year observation of hospital cases, Honduras. BMC Infectious Diseases. 2016;16:66. https://doi.org/10.1186/s12879-016-1393-6

12. World Health Organization. Bench Aids for the diagnosis of intestinal parasites. Second edition. Access: July 10, 2021. Available from: https://www.who.int/intestinal worms/ resources/9789241515344/en/

13. Osman M, El Safadi D, Cian A, Benamrouz S, Nourrisson C, Poirier P, et al. Prevalence and risk factors for intestinal protozoan infections with Cryptosporidium, Giardia, Blastocystis and Dientamoeba among schoolchildren in Tripoli, Lebanon. PLoS Negl Trop Dis. 2016;10:e0004496. https://doi.org/10.1371/journal.pntd.0004496

14. Almeria S, Cinar HN, Dubey JP. Cyclospora cayetanensis and Cyclosporiasis: An update. Microorganisms. 2019;7:317. https://doi.org/10.3390/microorganisms7090317

15. Gibney KB, O'Toole J, Sinclair M, Leder K. Burden of disease attributed to waterborne transmission of selected enteric pathogens, Australia, 2010. Am J Trop Med Hyg. 2017;96:1400-3. https://doi.org/10.4269/ajtmh.16-0907

16. Orozco-Mosqueda GE, Martínez-Loya O, Ortega Y. Cyclospora cayetanensis in a Pediatric Hospital in Morelia, México. Am J Trop Med Hyg. 2014;91:537-40. https://doi.org/10.4269/ajtmh.13-0535

17. Núñez FA, González OM, González I, Escobedo AA, Cordoví RA. Intestinal Coccidia in Cuban pediatric patients with diarrhea. Mem Inst Oswaldo Cruz. 2003;98:539-42. https://doi.org/10.1590/s0074-02762003000400021

18. Thivierge K, Iqbal A, Dixon B, Dion R, Levesque B, Cantin P, et al. Cryptosporidium hominis is a newly recognized pathogen in the Arctic Region of Nunavik, Canada: Molecular characterization of an outbreak. PLoS Negl Trop Dis. 2016;10:e0004534. https://doi.org/10.1371/journal.pntd.0004534

19. Dey A, Ghoshal U, Agarwal V, Ghoshal U. Genotyping of Cryptosporidium species and their clinical manifestations in patients with renal transplantation and human immunodeficiency virus infection. J Pathog. 2016;2016:2623602. https://doi.org/10.1155/2016/2623602

20. Mor SM, Tumwine JK, Ndeezi G, Srinivasan MG, Kaddu-Mulindwa DH, Tzipori S, et al. Respiratory cryptosporidiosis in HIV-seronegative children in Uganda: Potential for respiratory transmission. Clin Infect Dis. 2010;50:1366-72. https://doi.org/10.1086/652140

21. Mor SM, Ascolillo LR, Nakato R, Ndeezi G, Tumwine JK, Okwera A. Expectoration of Cryptosporidium parasites in sputum of human immunodeficiency virus-positive and negative adults. Am J Trop Med Hyg. 2018;98:1086-90. https://doi.org/10.4269/ajtmh.17-0741

22. Rivero-Rodríguez Z, Hernández A, Bracho A, Salazar S, Villalobos R. Prevalencia de microsporidios y otros enteroparásitos en pacientes con VIH positivo de Maracaibo, Venezuela. Biomédica. 2013;33:538-45. https://doi.org/10.7705/biomedica.v33i4.1468

23. Saramago-Peralta RH, Velásquez JN, de Souza-Cunha F, Pantano ML, Campos-Sodré F, da Silva S, et al. Genetic diversity of Cryptosporidium identified in clinical samples from cities in Brazil and Argentina. Mem Inst Oswaldo Cruz. 2016;111:30-6. https://doi.org/10.1590/0074-02760150303 
24. Yang Y, Zhou Y, Cheng W, Pan X, Xiao P, Shi Y, et al. Prevalence and determinants of Cryptosporidium infection in an underdeveloped rural region of Southwestern China. Am J Trop Med Hyg. 2017;96:595-601. https://doi.org/10.4269/ajtmh.16-0732

25. Shafiei R, Najjari M, Kargar-Kheirabad A, Hatam G. Severe diarrhea due to Cystoisospora belli infection in an HTLV-1 woman. Iran J Parasitol. 2016;11:121-5.

26. Chappell CL, Okhuysen PC, Langer-Curry RC, Lupo PJ, Widmer G, Tzipori S. Cryptosporidium muris: Infectivity and illness in healthy adult volunteers. Am J Trop Med Hyg. 2015;92:50-5. https://doi.org/10.4269/ajtmh.14-0525

27. Woon S, Yang R, Ryan U, Boan P, Prentice D. Chronic Cystoisospora belli infection in an immunocompetent Myanmar refugee -microscopy is not sensitive enough. BMC Infect Dis. 2016;16:221-5. https://doi.org/10.1186/s12879-016-1558-3

28. Golan-Shaposhnik E, Abozaid S, Grossman T, Marva E, On A, Azrad M, et al. The prevalence of Cryptosporidium among children hospitalized because of gastrointestinal symptoms and the efficiency of diagnostic methods for Cryptosporidium. Am J Trop Med Hyg. 2019;101:160-3. https://doi.org/10.4269/ajtmh.19-0057

29. Fleece ME, Heptinstall J, Khan S, Kabir M, Herbein J, Haque R, et al. Evaluation of a rapid lateral flow point-of-care test for detection of Cryptosporidium. Am J Trop Med Hyg. 2016;95:840-1. https://doi.org/10.4269/ajtmh.16-0132

30. Vieira-Silva C, Simão-Ferreira M, Gonçalves-Pires M, Costa-Cruz JM. Detection of Cryptosporidium - specific coproantigen in human immunodeficiency virus/acquired immunodeficiency syndrome patients by using a commercially available immunoenzymatic assay. Mem Inst Oswaldo Cruz. 2003;98:1097-9. https://doi.org/10.1590/s0074-02762003000800022

31. Ribeiro-Rolando R, da Silva S, Saramago-Peralta R, da Silva AJ, de Souza-Cunha F, Ribeiro-Bello A, et al. Detection and differentiation of Cryptosporidium by real-time polymerase chain reaction in stool samples from patients in Rio de Janeiro, Brazil. Mem Inst Oswaldo Cruz. 2012;107:476-9. https://doi.org/10.1590/s0074-02762012000400006 